\title{
An Interventional pilot project to expand the treatment facilities for asymptomatic COVID -19 patients by using the cost-effective strategy
}

\author{
Perera K.Y.D*, Dharmarathne G.S.K ${ }^{* *}$, Panapitiya P.W.C ${ }^{* *}$, Maddage A.K ${ }^{*}$, Perera U.A.A.S \\ * PGIM, UOC, Sri Lanka \\ ** Ministry of Health, Sri Lanka
}

DOI: 10.29322/IJSRP.11.01.2021.p10953

http://dx.doi.org/10.29322/IJSRP.11.01.2021.p10953

\begin{abstract}
The second wave of COVID-19 infection which resurged in October 2020, stroked Sri Lanka comparatively with a larger number of cases. To cater the increasing trend of COVID-19 positive cases, more primary and secondary healthcare institutions were converted into COVID-19 treatment and intermediate care centers

With the daily surge over 600-800 patients and the policy of institutional isolation of COVID patients, the health care administrators were compelled to find alternative ways to increase the total bed capacity in the country to manage it without compromising routine care services. Hence, this interventional project was carried out with the objective of expanding treatment facilities for asymptomatic COVID -19 patients. The project was implemented in three stages. Pre intervention assessment was carried out through key informant interviews with central and provincial level health authorities and reviewing of existing data to identify strategies. It was revealed that more than $80 \%$ of cases was asymptomatic who needs isolation and observation only. Accordingly, Temporary acquirement of the non-health government institutions to the Ministry of health and deploying of health services through nearby hospital were identified as strategies.

Acquisition of two youth centers with adequate space and converting them into intermediate centers with minimal temporary alteration were done under the guidance of technical expert. Material supply was accomplished using emergency procurement methods with the cost of one million rupees under the support of ADB and necessary labor supply was obtained from Sri Lanka Armed Forces. Health staff and logistics were deployed by nearby hospitals. Post interventional evaluation indicated that the project resulted in two exemplary low cost intermediate care centers within 10 days which contribute to COVID management in the country with a $97 \%$ bed occupancy rate.
\end{abstract}

Index Terms- Asymptomatic, Cost-effective, Intermediate- care, Treatment, COVID 19

Introduction

The second wave of COVID-19 infection which resurged in October 2020, stroked the Sri Lankan population comparatively in a large number of cases, unlike the first wave that occurred in mid-March 2020. Number of around 600-800 patients reported daily around the index case which was detected among garment factory workers in Gampaha, a district in the Western Province in the country.[1] As the daily surge of COVID positive patients influx exceeded the bed capacity of the previously designated six COVID 19 treatment hospitals, more and more primary and secondary healthcare institutions were decided to be converted into COVID-19 treatment centres. In this manner, the Ministry of Health was able to designate another 4000 beds to accommodate diagnosed COVID patients. [2]

Further, fourteen days of compulsory inward patient stay extended the length of inward stay of COVID confirmed patients. [3] This extended length of stay added another burden to the health care system of the country. As the pressure was building up, the health care administrators were compelled to find alternative ways to increase the total bed capacity of the country to manage the daily surge of patients.

All symptomatic patients and patients with comorbidities were admitted to designated COVID treatment centres under the observation of healthcare staff. However, the continuous on-demand conversion of primary and secondary healthcare facilities into treatment centres started affecting the routine healthcare services provided for patients with other ailments. Hence, the ministry of Health sought for an alternative solution with minimum impact on the provision of other health care. By the end of 2 weeks in October 2020, only $11 \%$ of allocated beds for patients with COVID-19 were available according to Medical Services Unit of Ministry of Health.[2]

Same time epidemiology data analysis of the disease revealed that around 80\% of the COVID patients of Sri Lanka are asymptomatic and do not require any specific treatment rather isolation to prevent the community spread. [1] Hence, the COVID expert committee of the Ministry of Health decided to expand the treatment facilities for asymptomatic COVID -19 patients by using a cost-effective strategy. 
This article describes the strategies taken by the ministry as a solution to expand the treatment facilities for asymptomatic COVID -19 patients by using the cost-effective strategy and managing the asymptomatic COVID 19 patients.

\section{Research Elaborations}

\section{Methodology \\ (Series of measures were taken to address the need to provide treatment centres for asymptomatic COVID patients at Gampaha district.)}

\section{A) Pre- Implementation Stage}

\section{At the national level}

Multiple key informant interviews with Secretary of Health, Additional Secretary of Health (Medical Services), Director General of Health Services, Deputy Director General / Medical Services and Director/ Healthcare Quality and Safety and Director/ Tertiary care services and economic analysis conducted by experts found that the construction of new hospitals are time-consuming and not costeffective. Then the Ministry of Health started to make partnerships with less utilized non-health government organizations to temporarily take over their spacious buildings and convert them as COVID-19 intermediate treatment centres for the asymptomatic COVID diagnosed patients with no medical comorbidities. Further, the administration of those intermediate care centres was transferred to the nearby secondary/tertiary care institutions. The project was initiated in October 2020, in two phases following reaching a consensus with the Health Department of the Western Province.

Phase 1. Temporary acquirement of the non-health government institutions to the Ministry of health and converting them into intermediate hospitals.

Phase 2. Transferring administration of converted hospitals and patient management to closest local hospitals of the Provincial Health Department.

For piloting this, the Ministry of Health took up two youth corps training centres at Yakkala and Wathupitiwala where then COVID19 hotspots. This decision was further discussed in the daily COVID - 19 review meeting chaired by the Secretary of the Ministry of Health and the green light was granted to go ahead in establishing intermediate hospitals for PCR tested positive patients.

\section{At regional Level}

Provincial Director of Health Services/ Western Province, Regional Director of Health Services/ Gampaha District, Directors/Medical Superintends of District General Hospital Gampaha and Base Hospital Wathupitiwala, Consultant Physicians, Consultant Respiratory Physicians, Medical Officer/ Public Health and Special Grade Nursing Officers along with administrative officers and accountants at institutional levels, conducted several meetings to develop operational and financial policies after which, following strategies were formulated.

I. Establishing a cost- effective intermediate care centres with 300 bed facilities

II. Providing clinical care through relevant consultants

III. Providing Human resources, supplies and logistics, supportive services like laundry and security through the nearby institutions.

To cope-up the objections and protests from local communities for establishing hospitals for COVID patients in their areas, Stakeholder meetings were arranged with local communities and mediated through religious leaders, politicians, and community leaders. All their concerns were discussed and educated on unclear areas of the COVID infection. With their requests, it was decided to provide special security services for these institutions to prevent patients from running away from these centres.

\section{B) Implementation Stage}

I. Asian Development Bank extended their generous support by funding the project. As a result of limited finances, it was decided to utilize the funds from Asian Development Bank worth SLR. 990,000.(SLR .99 million/US \$ 5350)

II. The Ministry of Health, in consultation with the clinicians and microbiologists and regional engineers, designed the structural layout and the construction were allocated to the Sri Lanka Air force constructing team as they have the capacity to do the structural changes in a fast-track way. Monitoring and supervision of the project was carried out by the experts of the Health Ministry.

III. Parallelly with the construction, the responsibility of the patient management was transferred to two closest local hospitals in the area. These hospitals were made responsible for deploying needed Human Resources, providing ancillary services, maintaining logistics supplies including health care waste management.

Iv. The formative and summative evaluation included the process evaluation of the project at different stages in view of completing it within 10 days, hence, the daily status of the project was evaluated. 


\section{C) Post Implementation Stage}

The successful completion of the project within 10 days had many impacts on the COVID patient management. Admitting patients to these centres prevented the patient load by preventing further community transferring of the disease. Furthermore, these two intermediate centres accommodated 300 asymptomatic COVID positive patients vacating 300 beds from COVID treatment hospitals enabling management more symptomatic patients in them.

Pre and post-intervention data from hospitals including routine statistics and situationally developed variables were used to measure the effects of the project. Number of patients treated at two centres, bed occupancy rate, number of patients infected with crossinfections, number of patients who developed COVID-19 related complications, and number of healthcare staff infected with COVID19 are the variables that were measured.

\section{Results or Finding}

Table 2; Allocated bed capacities at COVID centres by before and after the pilot project

\begin{tabular}{|l|l|l|l|}
\hline No. of treatment centres & No. of beds allocated & No. of beds occupied & \% of beds available \\
\hline Before the pilot project & 5400 & 4800 & 11.1 \\
\hline 48 & \multicolumn{3}{|l|}{} \\
\hline Immediately after the time of pilot project & $\begin{array}{l}4800 \\
\text { (by 4th week of October/2020) }\end{array}$ & 15.8 \\
\hline 50 & 5700 &
\end{tabular}

Table 3; Summative evaluation of newly constructed Intermediate care statistics by $4^{\text {th }}$ week of October 2020

\begin{tabular}{|l|l|}
\hline Indicator & Number/\% \\
\hline Number of patients treated at two centres & 1740 \\
\hline Bed occupancy rate & $96.6 \%$ \\
\hline Number of patients infected with cross-infections & 05 \\
\hline Number of healthcare staff infected with COVID 19 & 00 \\
\hline Number of patients had to isolated for more than 10-14 days due to cross-infections & 05 \\
\hline Number of patients transferred to tertiary care centres for managing complications & 04 \\
\hline
\end{tabular}

Patients' experience of COVID treatment centres, suggestions and comments collected at these intermediate hospitals were used in developing and improving the care of these centres. Over time, the patient-centered improvements increased the number of positive comments over the negative comments.

\section{Conclusions}

This project was the first of its kind in the country, which started as a pilot project during the peak of the second wave of COVID-19 pandemic in Sri Lanka. The Secretary of the Ministry of Health and the Director-General of Health Services used this project as a model in converting more and more non-health premises into centres accommodating asymptomatic patients. Further, it directly enabled managing patients in facilities which were close to their homeplace with greater convenience. Fewer transfers reduced the cost incurred on it.

Increasing the number of beds reduced patient congestion and likelihood of hospital-acquired infections, while improving, both patient as well as staff satisfaction in the treatment centres.

This project was a highly cost-effective project and was designed to work with limited available resources. The construction cost of the buildings was entirely saved along with the construction time. The conversion cost of 300 bedded hospitals was LKR 1 million. There was no additional cost incurred for employing health care staff and for administration. The health care staff were allocated on a roster basis from nearby hospitals, the administration was carried out by two designated hospitals. Task sharing and task shifting principles were used in places where appropriate in allocating health care staff. Essential patient monitoring equipment, beds either mobilized from nearby hospitals or received as donations. Additional costs incurred for meals, transport, and supportive care were reimbursed to the designated hospitals by the provincial authorities

REFERENCES
[1]Epidemiology Unit, $\quad$ Ministry $\quad$ of
https://www.epid.gov.lk/web/index.php?option=com content\&view=article\&id=225\&lang=en

[2] Director/ TCS, KII, Interviewed by Perera K.Y.D, 10th October 2020

[3] Health Alert, Ministry of Health, Sri Lanka, 2020., http://www.health.gov.lk/moh_final/english/heath_alert.php 


\section{AUTHORS}

First Author - Perera K.Y.D, MBBS, PGDME, PGDHQPS, PGDCH, MSc (Med. Admin), PGIM, UOC, Sri Lanka, dhanushkaperera@ymail.com

Second Author - Dharmarathne G.S.K, MBBS, MSc, MD (Med. Admin), Ministry of Health, Sri Lanka, sudathchamith@ gmail.com

Third Author - Panapitiya P.W.C, MBBS, MSc, MD (Med. Admin), Ministry of Health, Sri Lanka, lapnapitiya@ gmail.com

Fourth Author - Maddage A.K, MBBS, MSc, MD (Med. Admin), PGIM, UOC, Sri Lanka, krishanthmda@yahoo.com

Fifth Author - Perera U.A.A.S, MBBS, MSc, MD (Med. Admin), PGIM, UOC, Sri Lanka, anurasampath75@yahoo.com

Correspondence Author - Perera K.Y.D, MBBS, PGDME, PGDHQPS, PGDCH, MSc (Med. Admin), PGIM, UOC, Sri Lanka, dhanushkaperera@ymail.com, +94772647430 